\title{
INFLUÊNCIA DA CONSTANTE DE CORRSIN NO CÁLCULO DOS COEFICIENTES DE DIFUSÃO TURBULENTOS
}

\author{
Cristian Ricardo Nin Brauer \\ PPG em Física - CCNE \\ UFSM - Santa Maria, RS. \\ Gervásio Annes Degrazia \\ Departamento de Física - CCNE \\ UFSM - Santa Maria, RS. \\ Cláudia Rejane Jacondino de Campos \\ Faculdade de Meteorologia \\ UFPEL - Pelotas, RS.
}

\section{RESUMO}

Neste trabalho, expressões para os coeficientes de difusão turbulentos são obtidas usando diferentes valores da constante de Corrsin $(\gamma=0,44 ; \gamma=0,55$ e $\gamma=0,7)$. Estes coeficientes foram derivados da teoria de similaridade convectiva e do espectro turbulento unidimensional vertical válido em condições instáveis. Para testar a influência da constante de Corrsin no cálculo dos coeficientes de difusão turbulentos, foi empregado um modelo Euleriano de dispersão que permite a simulação da concentração de contaminantes e usa os coeficientes de difusão turbulentos aqui apresentados. As simulações de concentrações foram confrontadas com as concentrações observadas no experimento de Copenhagen. Os melhores resultados foram obtidos quando os valores de $\gamma=0,44$ e $\gamma=0,55$ foram utilizados no cálculo dos coeficientes de difusão turbulentos. 


\section{SUMMARY}

In this work, expressions for the eddy diffusivities are obtained using differents values from the Corrsin's constant $(\gamma=0,44 ; \gamma=0,55$ and $\gamma=$ $0,7)$. These eddy diffusivities are derived from the convective similarity theory and from the one dimensional vertical turbulent velocity spectrum, for unstable conditions. To test the influence of Corrsin's constant in the evaluation of the eddy diffusivities, was utilized an Eulerian dispersion model that permits the pollutants concentration simulation and uses the eddy diffusivities here presented. The simulations of the concentrations were confronted with the observed concentrations in the Copenhagen experiment. The best results were obtained when the values for the Corrsin's constant $\gamma=0,44$ and $\gamma=0,55$ are utilized in the eddy diffusivities.

\section{INTRODUÇÃO}

Nas últimas décadas, devido aos problemas ambientais causados pelo rápido desenvolvimento industrial e tecnológico, vem aumentando consideravelmente o estudo da dispersão e do transporte de poluentes na atmosfera. Conhecendo-se a estimativa do campo de concentração de contaminantes próximo à fonte é possível avaliar o impacto ambiental causado e agir no sentido de solucionar o problema de forma mais conveniente.

A equação da difusão-advecção tem sido frequentemente aplicada em modelos operacionais de dispersão de contaminantes atmosféricos para reproduzir o campo de concentração médio na Camada Limite Planetária (CLP). Em princípio, desta equação conhecendo-se o campo de velocidade médio e os fluxos turbulentos de concentração e dadas as condições iniciais e de contorno apropriadas, é possível obter um modelo teórico da dispersão de uma fonte pontual contínua. 
Muitas pesquisas em turbulência têm sido realizadas com o objetivo de especificar os fluxos turbulentos e resolver a equação da difusãoadvecção. O esquema mais utilizado de fechamento desta equação relaciona os fluxos turbulentos de concentração aos gradientes de concentração média através de coeficientes de difusão turbulentos.

Assim, para uma Camada Limite Convectiva (CLC) este trabalho tem por objetivo determinar a influência da constante de Corrsin, no cálculo dos coeficientes de difusão verticais turbulentos.

\section{COEFICIENTES DE DIFUSÃO TURBULENTOS PARA DIFERENTES VALORES DA CONSTANTE DE CORRSIN}

Os coeficientes de difusão turbulentos podem ser derivados a partir da teoria estatística de Taylor e do espectro de energia (Batchelor, 1949; Pasquill e Smith,1983 e Degrazia e Moraes, 1992) como segue:

$$
K_{\alpha}=\frac{\sigma_{i}^{2} \beta_{i}}{2 \pi} \int_{0}^{\infty} F_{i}(n) \frac{\sin \left(2 \pi n t / \beta_{i}\right)}{n} d n
$$

onde $\sigma_{i}^{2}$ é a variância da velocidade turbulenta, $\beta_{i}$ é um parâmetro que relaciona as escalas de tempo integral Lagrangeana e Euleriana, $n$ é a freqüência em Hertz ou ciclos/tempo, $t$ é o tempo de viagem, $F_{i}$ é o espectro de energia Euleriano, $i=u, \mathrm{v}, w$ e $\alpha=x, y, z$.

Estes coeficientes de difusão turbulentos, que dependem da distância da fonte, permitem capturar o efeito de memória, que está presente no campo turbulento próximo à fontes pontuais elevadas.

Para grandes tempos de viagem o coeficiente de difusão turbulento na equação (1) torna-se independente da distância da fonte e é função apenas das propriedades locais da turbulência. Neste caso, o 
coeficiente de difusão turbulento válido para uma fonte de área infinita na superfície, é escrito como segue:

$$
K_{\alpha}=\frac{\sigma_{i}^{2} \beta_{i} F_{i}(0)}{4} .
$$

Nas equações (1) e (2) a dinâmica da dispersão está relacionada com os turbilhões que contém energia e com as escalas de similaridade fundamentais que os descrevem.

Neste trabalho, estamos interessados em calcular os coeficientes de difusão turbulentos para uma CLC seguindo este propósito, é necessário definir a variância da velocidade turbulenta $\sigma_{i}{ }^{2}$, o espectro de energia Euleriano $F_{i}$ e o fator de escala $\beta_{i}$ para condições instáveis.

O espectro de velocidade para condições instáveis pode ser expresso como uma função de escalas convectivas (Degrazia et al., 1996), como segue:

$$
\frac{n S_{i}^{E}(n)}{w_{*}^{2}}=\frac{1,06 c_{i}(f / q)(\psi / q)^{2 / 3}\left(z / z_{i}\right)^{2 / 3}}{\left(f_{m}\right)_{i}^{5 / 3}\left[1+\frac{1,5}{\left(f_{m}\right)_{i}} \frac{f}{q}\right]^{5 / 3}}
$$

onde:

$S_{i}^{E}$ é a função densidade espectral de energia turbulenta Euleriana, $n$ é a freqüência em Hertz ou ciclos/tempo, $w_{*}$ é a escala de velocidade na CLC, $c_{i}$ é uma constante numérica, $f$ é a freqüência adimensional, $q$ é o parâmetro de estabilidade, $\psi$ é a função de dissipação adimensional, $z$ é a altura acima do solo, $z_{i}$ é a altura da CLC e $f_{m}$ é a freqüência adimensional associada ao máximo do espectro. 
A integração analítica de $S_{i}^{E}$ na equação (3) sobre todo o domínio de freqüência fornece a variância da velocidade turbulenta:

$$
\sigma_{i}^{2}=w_{*}^{2} \frac{1,06 c_{i}}{\left(f_{m}\right)_{i}^{2 / 3}}\left(\frac{\psi}{q}\right)^{2 / 3}\left(\frac{z}{z_{i}}\right)^{2 / 3} .
$$

Essa variância é usada para normalizar o espectro de energia Euleriano, que é então dado por:

$$
F_{i}(n)=\frac{n S_{i}^{E}(n)}{\sigma_{i}^{2}}=\frac{1}{\left(f_{m}\right)_{i}} \frac{f}{q}\left[1+\frac{1,5}{\left(f_{m}\right)_{i}} \frac{f}{q}\right]^{-5 / 3}
$$

para $n=0$

$$
F_{i}(0)=\frac{S_{i}^{E}(0)}{\sigma_{i}^{2}}=\frac{1}{\left(f_{m}\right)_{i}} \frac{z}{U q} .
$$

Finalmente o fator de escala $\beta_{i}$ pode ser expresso como (Wandel e Kofoed, 1962; Angell, 1974; Pasquill, 1974; Hanna, 1981)

$$
\beta_{i}=\frac{T_{L_{i}}}{T_{i}}=\gamma \frac{U}{\sigma_{i}}
$$

onde $T_{L_{i}}$ é a escala de tempo integral Lagrangeana, $T_{i}$ é a escala de tempo integral Euleriana, $U$ é a velocidade do vento médio e $\gamma$ é a constante de Corrsin. Esta constante assume vários valores na literatura, já que ela não é uma constante universal, mas depende do tipo de fluxo turbulento (Hinze, 1975). Neste trabalho, para testar a sua influência no cálculo dos coeficientes de difusão turbulentos, foram utilizados três valores: i) $\gamma=0,44$, por ser o valor teórico mais utilizado (Wandel e Kofoed, 1962; Corrsin, 1963), ii) $\gamma=0,55$, por ser o valor médio de uma série de trabalhos teóricos e experimentais 
(Degrazia e Anfossi, 1998) e iii) $\gamma=0,7$, por ser o último valor experimental obtido para condições instáveis (Hanna, 1981).

Agora, substituindo-se as equações (4), (6) e (7) na equação (2) e assumindo $\gamma=0,44 ; \gamma=0,55$ e $\gamma=0,7$ tem-se respectivamente:

$$
\begin{aligned}
& K_{\alpha}=\frac{0,44}{4} \frac{\sigma_{i} z}{\left(f_{m}\right)_{i} q} \\
& K_{\alpha}=\frac{0,55}{4} \frac{\sigma_{i} z}{\left(f_{m}\right)_{i} q} \\
& K_{\alpha}=\frac{0,7}{4} \frac{\sigma_{i} z}{\left(f_{m}\right)_{i} q} .
\end{aligned}
$$

Por considerar homogeneidade horizontal do campo turbulento, a evolução da CLC é controlada principalmente pelo transporte vertical de calor. Portanto, a análise a partir de agora é focalizada sobre o coeficiente de difusão turbulento vertical.

Então, assumindo $\left(f_{m}\right)_{w}=0,33$ (Sorbjan, 1986);

$q=\frac{z}{\left(f_{m}\right)_{w}\left(\lambda_{m}\right)_{w}}=1,68\left(\frac{z}{z_{i}}\right)\left[1-\exp \left(-4 \frac{z}{z_{i}}\right)-0,0003 \exp \left(8 \frac{z}{z_{i}}\right)\right]^{-1}$

(Caughey e Palmer, 1979)

e

$$
\sigma_{w}=1,08\left(\frac{z}{z_{i}}\right)^{1 / 3}\left(1-\frac{z}{z_{i}}\right)^{1 / 3} w_{*} \quad \text { (Sorbjan, 1989), }
$$

os coeficientes de difusão turbulentos, equações (8), (9) e (10) podem ser escritos respectivamente como:

$$
\frac{K_{z}}{w_{*} z_{i}}=0,22\left(\frac{z}{z_{i}}\right)^{1 / 3}\left(1-\frac{z}{z_{i}}\right)^{1 / 3}\left[1-\exp \left(-4 \frac{z}{z_{i}}\right)-0,0003 \exp \left(8 \frac{z}{z_{i}}\right)\right],
$$

84 Rev. Ciência e Natura, Dispersion Process: 79 - 91 , 2000. 


$$
\begin{aligned}
& \frac{K_{z}}{w_{*} z_{i}}=0,27\left(\frac{z}{z_{i}}\right)^{1 / 3}\left(1-\frac{z}{z_{i}}\right)^{1 / 3}\left[1-\exp \left(-4 \frac{z}{z_{i}}\right)-0,0003 \exp \left(8 \frac{z}{z_{i}}\right)\right] \\
& \frac{K_{z}}{w_{*} z_{i}}=0,34\left(\frac{z}{z_{i}}\right)^{1 / 3}\left(1-\frac{z}{z_{i}}\right)^{1 / 3}\left[1-\exp \left(-4 \frac{z}{z_{i}}\right)-0,0003 \exp \left(8 \frac{z}{z_{i}}\right)\right]
\end{aligned}
$$

\section{TESTE DA INFLUÊNCIA DOS VALORES DA CONSTANTE DE CORRSIN NA DISPERSÃO DE CONTAMINANTES}

Para testar a influência da constante de Corrsin no cálculo do coeficientes verticais de difusão turbulentos, utilizou-se o modelo Euleriano de dispersão proposto por Vilhena et al. (1998).

Este modelo calcula a concentração integrada lateral $\left(c^{y}\right)$ de contaminantes, ao nível do solo emitida por uma fonte elevada. Foram feitas três simulações com este modelo, levando em conta os três coeficientes de difusão turbulentos propostos nas equações (11), (12) e (13). Na tabela 1, são apresentados os resultados das simulações $e$ os dados de concentrações de contaminantes medidos ao nível do solo, usando o traçador $\mathrm{SF}_{6}$ durante o experimento de Copenhagen que ocorreu em condições atmosféricas de instabilidade moderada (Gryning e Lyck, 1984). O traçador utilizado no experimento foi liberado sem empuxo de uma fonte com altura de 
$115 \mathrm{~m}$ e coletado ao nível do solo em três arcos perpendiculares à direção do vento médio. Os arcos foram posicionados de 2 a $6 \mathrm{Km}$ do ponto no qual ocorreu a liberação do traçador. O sítio experimental era residencial com um parâmetro de rugosidade de $0,6 \mathrm{~m}$.

Tabela 1: Concentrações integradas laterais observadas $\left(\overline{c^{y}}\right)$ e simuladas usando o conjunto de dados de Copenhagen. $\left(\overline{c^{y}}\right)_{\gamma=0,44},\left(\overline{c^{y}}\right)_{\gamma=0,55}$ e

\begin{tabular}{cccccc}
\multicolumn{7}{c}{$\left(\overline{c^{y}}\right)_{\gamma=0,7} \cdot$} & \multicolumn{3}{c}{} \\
\hline \multirow{2}{*}{ Exp. } & $\begin{array}{c}\text { Distância } \\
(\mathrm{m})\end{array}$ & $\begin{array}{c}\left(\overline{c^{y}}\right) \\
\left(10^{-4} \mathrm{~s} / \mathrm{m}^{2}\right)\end{array}$ & $\begin{array}{c}\left(\overline{c^{y}}\right)_{\gamma=0,44} \\
\left(10^{-4} \mathrm{~s} / \mathrm{m}^{2}\right)\end{array}$ & $\begin{array}{c}\left(\overline{c^{y}}\right)_{\gamma=0,55} \\
\left(10^{-4} \mathrm{~s} / \mathrm{m}^{2}\right)\end{array}$ & $\left(\overline{c^{y}}\right)_{\gamma=0,7}$ \\
$\left(10^{-4} \mathrm{~s} / \mathrm{m}^{2}\right)$
\end{tabular}

86 Rev. Ciência e Natura, Dispersion Process: 79 - 91 , 2000. 
A análise da tabela 1 mostra que as concentrações de contaminantes simuladas pelo modelo de Vilhena et al. (1998) são próximas as concentrações observadas em Copenhagen.

Para uma análise mais precisa destes resultados, um confronto entre as simulações e as observações foi realizado utilizando um conjunto de índices estatísticos (Hanna, 1989), definidos como segue:

Erro quadrático médio normalizado $($ nmse $)=\frac{\overline{\left(C_{o}-C_{p}\right)^{2}}}{\overline{C_{o} C_{p}}}$ : informa sobre todos os desvios entre concentrações dos modelos e dos dados observados. É uma estatística adimensional e seu valor deve ser o menor possível para se ter um bom modelo.

Coeficiente de correlação cor $=\frac{\overline{\left(C_{o}-\overline{C_{o}}\right)\left(C_{p}-\overline{C_{p}}\right)}}{\sigma_{o} \sigma_{p}}$ : descreve o grau de associação ou concordância entre as variáveis. Para uma boa performance o seu valor deve ser próximo de 1.

Fator de dois fa2 = fração de dados para os quais $(\%)$

$0,5 \leq \frac{C_{p}}{C_{o}} \leq 2$, um valor ótimo é 1.
Desvio fracional $f b=\frac{\overline{C_{o}}-\overline{C_{p}}}{0,5\left(\overline{C_{o}}+\overline{C_{p}}\right)}$ : informa a tendência do modelo de sobrestimar ou subestimar as concentrações observadas. $\mathrm{O}$ valor ótimo é zero.

Desvio fracional padrão $f_{s}=2 \frac{\sigma_{o}-\sigma_{p}}{\sigma_{0}+\sigma_{p}}$, o valor ótimo é zero.

Os índices o e p indicam respectivamente, as quantidades observadas e preditas. 
A análise estatística utilizando os dados de todos os experimentos ( 1 a 9 ) de Copenhagen e somente aqueles mais convectivos (experimentos 1, 3, 7 e 8) é apresentada, respectivamente, nas tabelas 2 e 3 .

Tabela 2: Índices estatísticos para todos os dados de Copenhagen

\begin{tabular}{lllllll}
\hline modelo & Bias & nmse & cor & fa2 & fb & Fs \\
\hline$\gamma=0,44$ & $-0,20$ & 0,05 & 0,917 & 1,000 & 0,045 & 0,211 \\
$\gamma=0,55$ & $-0,34$ & 0,06 & 0,915 & 1,000 & 0,079 & 0,203 \\
$\gamma=0,7$ & $-1,15$ & 0,16 & 0,919 & 1,000 & 0,293 & 0,336 \\
\hline
\end{tabular}

Tabela 3: Índices estatísticos para os casos mais convectivos ( experimentos

\begin{tabular}{lllllll}
\multicolumn{7}{c}{$1,3,7$ e 8$)$ : } \\
\hline modelo & bias & nmse & cor & fa2 & fb & Fs \\
\hline$\gamma=0,44$ & 0,05 & 0,06 & 0,870 & 1,000 & $-0,10$ & 0,181 \\
$\gamma=0,55$ & $-0,17$ & 0,06 & 0,871 & 1,000 & 0,041 & 0,222 \\
$\gamma=0,7$ & $-1,15$ & 0,19 & 0,872 & 1,000 & 0,300 & 0,473 \\
\hline
\end{tabular}

A análise estatística, apresentada na tabela 2, mostra que os melhores resultados simulados pelo modelo foram gerados quando os valores de $\gamma=0,44$ e $\gamma=0,55$ foram utilizados no cálculo dos coeficientes de difusão turbulentos.

A tabela 3 sugere que mesmo em condições instáveis $\gamma=0,44$ e $\gamma=0,55$ continuam sendo os valores mais adequados na construção de coeficientes de difusão turbulentos.

A presente análise indica que a escolha de $\gamma=0,44$ e $\gamma=0,55$ conduz a uma simulação mais realista das concentrações observadas. 88 Rev. Ciência e Natura, Dispersion Process: 79 - 91 , 2000. 


\section{CONCLUSÕES}

Neste trabalho foi testada a influência da constante de Corrsin no cálculo dos coeficientes de difusão turbulentos na CLC. Para tal, utilizouse o modelo Euleriano de dispersão proposto por Vilhena et al. (1998).

Foram geradas três simulações: uma com $\gamma=0,44$, outra com $\gamma=0,55$ e uma última com $\gamma=0,7$. Os resultados das três simulações foram confrontados com as observações de Copenhagen e mostraram que mesmo em condições bem instáveis(convectivas) $\gamma=0,44$ e $\gamma=0,55$ são os valores mais adequados na construção de coeficientes de difusão turbulentos.

Sugere-se que, para uma maior confiabilidade dos resultados aqui apresentados, a análise aqui desenvolvida seja estendida para um número maior de experimentos de dispersão.

\section{AGRADECIMENTOS}

Trabalho parcialmente financiado pelo Conselho Nacional de Desenvolvimento Científico e Tecnológico (CNPq) e pela Fundação de Amparo a Pesquisa do Rio Grande do Sul (FAPERGS). 


\section{BIBLIOGRAFIA}

ANGEL, J. K.: Lagrangian-Eulerian time-scale relationship estimated from constant volume ballon flights past a tall tower, Advances in Geophysics, 18 A, Academic Press, 419-432. 1974

BATCHELOR, G. K.: Diffusion in a Field of Homogeneous Turbulence, I: Eulerian Analysis, Aust. J. Sci. Res. 2, 437-450. 1949

CAUGHEY, S. J. AND PALMER, S. G.: Some aspects of turbulence structure through the depth of the convective boundary layer, Q. J. R. Meteorol. Soc., 105, 811-827. 1979

CORRSIN, S.: Estimates of the relations between Eulerian and Lagrangian scales in large Reynolds Number turbulence, Journal of Atmospheric Science, 20, 115-119. 1963

DEGRAZIA AND MORAES, O. L. L.: A Model for Eddy Diffusivity in a Stable Boundary Layer, Boundary-Layer Meteorol., 58, 205-214. 1992 , MORAES, O. L. L. AND OLIVEIRA, A. P.: An Analitycal Method to Evaluate Mixing Length Scales for the Planetary Boundary Layer, J. Appl. Meteorol., 35, 974-977. 1996

AND ANFOSSI, D.: Estimation of the Kolmogorov constant $\mathrm{C}_{0}$ from classical statistical diffusion theory, Atmos. Environm., 32, 3611-3614. 1998

GRYNING, S. W. AND LYCK, E.: Atmospheric dispersion from elevated sources in an urban area: comparison between tracer experiments and model calculations, J. Climate Appl. Meteor., 23, 651-660. 1984

HANNA, S. R.: Lagrangian and Eulerian Time-Scale in the Daytime Boundary Layer, J. Appl. Meteorol. 20, 242-249. 1981

: Confidence limit for air quality models as estimated by bootstrap and jacknife resampling methods, Atmos. Environ., 23, 1385-1395. 1989 HINZE, J. O.: Turbulence, Mc Graw Hill, 790 pp.. 1975

PASQUILL, F.: Atmospheric Diffusion, Wiley \& Sons, 429 pp.. 1974 
, AND SMITH, F. B.: Atmospheric Diffusion, Wiley \& Sons, 437 pp.. 1983

, AND SMITH, F. B.: Atmospheric diffusion, Ellis Horwood Ltd., Chichester, 437pp. 1983

SORBJAN, Z.: Local similarity of spectral and cospectral characteristics in the stable boundary layer, Boundary-Layer Metorol., 35, 257-275. 1986 : Stucture of the Atmospheric Boundary Layer, Prentice Hall, NJ, 317 pp.. 1989

VILHENA, M. T., RIZZA, U., DEGRAZIA, G. A., MANGIA, C., MOREIRA, D. M. AND TIRABASSI, T.: An Analytical Air Pollution Model: Development and Evaluation, Contr. Atmos. Phys., 71, 315-320. 1998

WANDEL, C. F. AND KOFOED-HANSEN, O.: On the Eulerian-Lagrangian transform in the statistical theory of turbulence, J. Geophys. Res., 76, 3089-3093. 1962 
92 Rev. Ciência e Natura, Dispersion Process: 79 - 91 , 2000. 